emanzipiert hatten (Sri Lanka) oder zu emanzipieren begannen (immerhin mit indischer Duldung) (Nepal) als die Bedürfnisse virulent wurden (nach 1962 bzw. 1967).

Insgesamt ist das Buch freilich eine lohnende Lektüre, da hier erstmals im deutschen Sprachraum die Außen- und Wirtschaftsbeziehungen Indiens systematisch aufgearbeitet und unter einem neuen theoretischen Blickwinkel betrachtet wurden.

Joachim Betz

Brian E. McKnight (Ubers.)

The Washing Away of Wrongs: Forensic Medicine in Thirteenth-Century China, Ann Arbor, Center for Chinese Studies, The University of Michigan, 1981, XV + 181 S. (Science, Medicine, and Technology in East Asia, Vol. 1)

Unsere Kenntnisse von den Rechtsverhältnissen im älteren China haben sich nicht zuletzt dank der Úbersetzung von Gesetzestexten, juristischen Handbüchern und Fallsammlungen beträchtlich erweitert. Neue aus dem Ende des 3. Jahrhunderts v. Chr. stammende Textfunde belegen schließlich, daß bestimmte Rechtstraditionen in China älter sind als bisher vermutet. So gibt es heute auch Belege für eine etablierte Praxis einer Leichenschau unter forensischen Gesichtspunkten bereits unter der Dynastie Ch'in (reg. 221-207 v. Chr.), von denen ein Beispiel kürzlich Katrina C. D. McLeod und Robin D. S. Yates in ihrem Artikel "Forms of Ch'in Law: An Annotated Translation of the Feng-chen shih" (Harvard Journal of Asiatic Studies 41:1 (June 1981) S. 111-163, bes. S. $154 \mathrm{f}$.) vorgestellt haben. (Vgl. a. Derk Bodde, Forensic Medicine in Pre-Imperial China, in: Journal of the American Oriental Society vol. 102 (1982) 1-15.) Zur Zeit der Sung-Dynastie (960-1279) wurden jedoch offenbar die Vorschriften zur amtlichen Untersuchung bei Fällen unnatürlichen Todes neu gefaßt, und man kann vermuten, daß die Vorschriften dank der Blockdrucktechnik in Form von Handbüchern weite Verbreitung fanden. Das älteste erhaltene und bis in neuere Zeit in Ostasien in verschiedenen Fassungen sehr verbreitete Vorschriften zum Verfahren sowie das Grundwissen zur Durchführung der amtlichen Leichenbeschau enthaltende Handbuch ist das Hsi yüan chi lu ("Collected Writings on the Washing Away of Wrongs") des Sung Tz'u (1186-1249 n. Ch.) a us dem Jahre 1247, das Prof. McKnight nun in neuer und erstmals vollständiger englischer Ubersetzung vorgelegt hat. Beachtung hat dieses in der in den letzten Jahren auch in China wieder verstärkt betriebenen Wissenschaftsgeschichte als "Meilenstein" angesehene Werk bereits seit langem im Westen gefunden. Doch basieren die bisherigen Ubersetzungen auf späteren Fassungen und sind bis auf diejenigen des Holländers C. F. M. deGrijs aus dem Jahre 1863, die von Heinrich Breitenstein (Gerichtliche Medizin der Chinesen, Leipzig 1908) und diejenige des Engländers Herbert Giles aus dem Jahre 1874 (in Band 3 von China Review) nur Teilübersetzungen (s. S. 31 f.). Da die auch den "vollständigen " Úbersetzungen zugrundeliegenden Fassungen nur etwa zur Hälfte mit 
dem aus der Yüan-Dynastie (1280-1367) überlieferten Text übereinstimmen, ist die neue Ubersetzung McKnights ein wichtiger Beitrag zu unserer Kenntnis und zur weiteren Erforschung ebenso der forensischen Medizin wie der Verwaltungspraxis auf unterer Ebene, einem Gebiet, auf dem McKnight bereits grundlegende Forschungsarbeit geleistet hat (Village and Bureaucracy in Southern Sung China, Chicago: University of Chicago Press 1971). Mit dieser Ubersetzung, in der McKnight nur die sungzeitlichen Teile der in der Sammlung Tai-nan ko ts'ung-shu überlieferten yüanzeitlichen Version vorlegt (die Paginierung der von ihm benutzten Ausgabe des Textes, Peking 1953, stimmt nicht mit der eher als Standard anzunehmenden Paginierung des Nachdruckes des Tai-nan ko ts'ung-shu im Pai-pu ts'ung-shu chi-ch'eng überein), ist der weiteren Erforschung des Themas eine wichtige Hilfe gegeben. Gleichwohl bleiben, worauf auch Peter Kees Bol in seiner Besprechung im Journal of Asian Studies, vol. XLII, 3 (May 1983), S. 643 f., hingewiesen hat, zahlreiche Fragen hinsichtlich der Tradition, in der Sung Tz'u steht bzw. von der er sich abhebt ebenso wie hinsichtlich des Textverständnisses und der Terminologie offen. Leider hat McKnight nicht mehr die von Yang Feng-k'un annotierte und mit umgangssprachlicher Ubersetzung versehene Ausgabe des Textes Hsi yüan chi lu chiao i, Peking 1980, heranziehen können. Der Ubersetzung hat McKnight eine sehr brauchbare Einleitung vorangestellt (S. 2-34), in der er auch einige komparatistische Betrachtungen anstellt sowie Auskünfte über den Autor, Sung Tz'u, und frühere Ubersetzungen, wie über die Schwierigkeiten beim Ubersetzen gibt. Eine strukturelle Analyse des Handbuches, in der auch im Hinblick auf sonstige Handbücher Aufbau und Gliederung dargestellt werden müßten, hat McKnight leider nicht versucht. Dies wie auch die Erörterung anderer Fragen steht noch aus. Die neue Úbersetzung McKnights stellt einen Ansporn, jedenfalls aber eine Hilfe dar und einen Beitrag zur weiteren Beschäftigung mit diesem Text, der für die historische Rechtsvergleichung und die Geschichte der Medizin ebenso von zentraler Bedeutung ist wie für das bessere Verständnis der Wissenschaften und der Verwaltungspraxis im vormodernen China.

Helwig Schmidt-Glintzer

\section{Madhu Sarin}

Urban Planning in the Third World. The Chandigarh Experience,

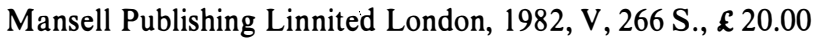

"New Towns", d. h. Stadtneugründungen, die auf dem Reißbrett entstanden sind, werden in vielen Ländern der Dritten Welt nicht nur als Ausweg aus den drückenden Problemen städtischer Unterentwicklung, sondern häufig auch als nationale Fortschrittssymbole betrachtet. Unter den bekanntesten "New Towns" - zu nennen wären hier etwa Tema (Ghana), Brasilia (Brasilien), Ciudad Guayana (Venezuela), Nasser City (Ägypten), Islamabad (Pakistan) oder Dodoma (Tanzania) - wird der indischen Stadt 